\title{
Ultrasonographic assessment of neuromuscular electrical stimulation efficacy on glenohumeral subluxation in patients with hemiplegia: a randomized-controlled study
}

\author{
Canan Türkkan, ${ }^{1}$ Gökhan Tuna Öztürk, ${ }^{1}$ Fatma Gülçin Uğurlu, ${ }^{2}$ Murat Ersöz ${ }^{1}$ \\ ${ }^{1}$ Department of Physical Medicine and Rehabilitation, Ankara Physical Medicine and Rehabilitation Training and Research Hospital, Ankara, Turkey \\ ${ }^{2}$ Department of Physical Medicine and Rehabilitation, Medical Faculty of Ylldırım Beyazit University, Ankara, Turkey
}

Received: October 2016 Accepted: February 2017

\begin{abstract}
Objectives: This study aims to investigate the short-term effects of neuromuscular electrical stimulation (NMES) on glenohumeral subluxation (GHS) in stroke patients.

Patients and methods: This prospective, randomized-controlled study included 24 unilateral hemiplegic patients (10 males, 14 females; mean age $64.1 \pm 14.8$ years; range 22 to 84 years) with GHS as assessed by ultrasonography between December 2013 and September 2014 . The patients were randomly divided into two groups as those in the NMES group $(n=12)$ who were treated with NMES to supraspinatus, upper trapezius, and posterior deltoid muscles combined with conventional physiotherapy and as those in the control group ( $\mathrm{n}=12$ ) who were received conventional physiotherapy alone. Clinical (the Brunnstrom Motor Recovery Stage, Visual Analog Scale [VAS] for Pain and Shoulder Disability Questionnaire [SDQ]) and ultrasonographic (acromion-greater tuberosity distance, thicknesses of supraspinatus, upper trapezius, and posterior deltoid muscles) variables were evaluated before and after treatment in both groups.

Results: The SDQ index, acromion-greater tuberosity distance, and supraspinatus muscle thickness were improved in the NMES group, compared to the control group (for all $\mathrm{p}<0.05$ ). The VAS-pain scores decreased in both groups. There was no statistically significant alterations in the other measurements in both groups (for all $\mathrm{p}>0.05$ ). The percentage change (\%) of the VAS-pain scores was not significantly different between two groups ( $\mathrm{p}=0.03)$.
\end{abstract}

Conclusion: Our study results showed that GHS decreased after 20 sessions of NMES treatment. Based on these findings, ultrasonography appears to be a proper imaging tool for the evaluation of GHS in stroke patients.

Keywords: Electrical stimulation; glenohumeral subluxation; ultrasonography.

Glenohumeral subluxation (GHS) is described as an increased translation of the humeral head relative to the glenoid fossa, and is usually seen within the three weeks after stroke. ${ }^{[1]}$ It mostly occurs in hemiplegic patients as an inferior subluxation with an incidence of 17 to $81 \% \cdot{ }^{[2-4]}$ Supraspinatus and posterior deltoid muscles contribute to the shoulder stabilization, and they prevent the inferior subluxation. ${ }^{[1]}$ Severe impairment of motor function, particularly in these muscles, after stroke is a potential risk factor for subluxation. ${ }^{[5]}$ Shoulder subluxation interferes with the daily life activities due to impaired upper limb motor function, which may prolong the rehabilitation process. Therefore, it is important for the physician to detect the presence of GHS and to tailor the prompt treatment immediately.

Various clinical and radiographic methods have been described for the evaluation of GHS. ${ }^{[6-10]}$ Current clinical methods include palpation, the number of finger-breaths, and use of thermoplastic jigs. However, these clinical methods are physician-dependent with low sensitivities. ${ }^{[6,7]}$ Qualitative and quantitative radiographs are also useful to obtain an objective evaluation. Although radiographs have high reliability and validity, they have some disadvantages such as high cost, and being time-consuming and radiation

Corresponding author: Canan Türkkan, MD. Ankara Fiziksel Tıp ve Rehabilitasyon Eŭitim ve Araştırma Hastanesi Fiziksel Tıp ve Rehabilitasyon Kliniği, 06230 Sihhiye, Ankara, Turkey. e-mail: drcananpolat@gmail.com 
exposure. ${ }^{[8-11]}$ On the other hand, musculoskeletal ultrasonography (USG) has several advantages (i.e., inexpensive, convenient, dynamic, radiation-free) over direct radiographic imaging, and it is widely used for musculoskeletal conditions, particularly in the shoulder region. ${ }^{[12,13]}$ Furthermore, it has been shown to be a valid and reliable method for the assessment of the GHS in post-stroke patients. ${ }^{[14]}$

Treatment of shoulder subluxation includes the use of shoulder supports and neuromuscular electrical stimulation (NMES). Several studies have shown that NMES application on supraspinatus and posterior deltoid muscles is an effective treatment method for GHS. ${ }^{[15,16]}$ Additionally, positive effects of NMES on shoulder subluxation were shown through radiographic imaging. ${ }^{[17]}$ However, to the best of our knowledge, the effects of NMES on GHS using USG have not been studied in stroke patients previously. Therefore, in the present study, we aimed to investigate the NMES effects on shoulder subluxation in stroke patients through USG.

\section{PATIENTS AND METHODS}

Twenty-four patients with hemiplegia who were rehabilitated at Ankara Physical Medicine and Rehabilitation Training and Research Hospital Rehabilitation Center between December 2013 and September 2014 were included in this prospective, randomized-controlled study. All stroke patients with GHS were included in the study. Patients with severe heart failure, bilateral hemiplegia, or other shoulder pathologies were excluded. The approval of study protocol was obtained from the Ankara Physical Medicine and Rehabilitation Training and Research Hospital Ethics Committee and a written informed consent was obtained from each participant. The study was conducted in accordance with the principles of the Declaration of Helsinki.

The patients $(\mathrm{n}=24)$ were randomly divided into two groups as the NMES group $(n=12)$ and control group $(n=12)$. Each patient was numbered according to the order of admission. These numbers were randomly distributed into two groups using a computer program. All patients used a shoulder strap and received similar conventional physiotherapy for GHS (range of motion, stretching, and strengthening exercises). Additionally, in the NMES group, the patients received NMES treatment. Conventional physiotherapy was performed by a physiotherapist in the rehabilitation center, while NMES was applied by the physiartrist. Also, NMES was applied to supraspinatus, upper trapezius, and posterior deltoid muscles of the hemiplegic side for $60 \mathrm{~min} / \mathrm{session}$ in a day, and five days a week for four weeks (a total of 20 sessions). The patients were held in the sitting position (shoulder neutral position, elbow flexed $90^{\circ}$, forearm in pronation), and a twochannel multimodal electrostimulator (SAMMS Mod Professional, Chattanooga Group Inc. USA) which has four surface electrodes with the size of $5.5 \times 6.5 \mathrm{~cm}$, were used. For supraspinatus and upper trapezius stimulation, the active electrode was placed on $5 \mathrm{~cm}$ away from the acromion at the level of the midpoint of the scapular spine. For stimulation of posterior deltoid muscle, the active electrode was placed on $5 \mathrm{~cm}$ distal of the posterior acromion. The intensity of electrical stimulation was administered in the range from 20 to $30 \mathrm{~mA}$ (frequency was $25 \mathrm{~Hz}$, sequence pulse width was $250 \mu \mathrm{s})$. The stimulation intensity was progressively increased, until contraction was obtained based on the tolerance of each patient.

Demographic features of patients including age, Body Mass Index, hemiplegic side, and duration of stroke were recorded. Before and after treatment, the affected upper extremity functions were evaluated using the Brunnstrom Motor Recovery Stage (BMRS), which is a valid and reliable method for assessing motor functions consisting of six stages: (i) flaccid paralysis; (ii) involuntary movement and spasticity in synergy pattern; (iii) increased spasticity and voluntary control in synergy pattern; (iv) spasticity declines, voluntary movement without synergy pattern; (v) spasticity becomes small, more complex movements are observed; and (vi) spasticity disappears, normal movements are observed. ${ }^{[18,19]}$ Shoulder functional status was measured using the Shoulder Disability Questionnaire (SDQ), which includes 16 questions describing common conditions which may induce symptoms in patients with shoulder disorders. ${ }^{[20]}$ Each item is evaluated for the past 24 hours, and answer options are yes, no, or not applicable, and a final score range is between 0 (no disability) and 100 (all applicable items positive). The shoulder pain was evaluated using the $0-100 \mathrm{~mm}$ Visual Analog Scale for Pain (VAS-pain).

Ultrasonographic evaluations were done using a 5-12 MHz linear array probe (Logiq P5, General Electric, Wisconsin, USA). The patients were seated with the shoulder in neutral rotation and the elbow at $90^{\circ}$ of flexion and forearm in pronation. Acromion-greater tuberosity (A-GT) distance and thicknesses of supraspinatus, upper trapezius, and posterior deltoid muscles were measured. For the A-GT distance measurements, the lateral edge of 

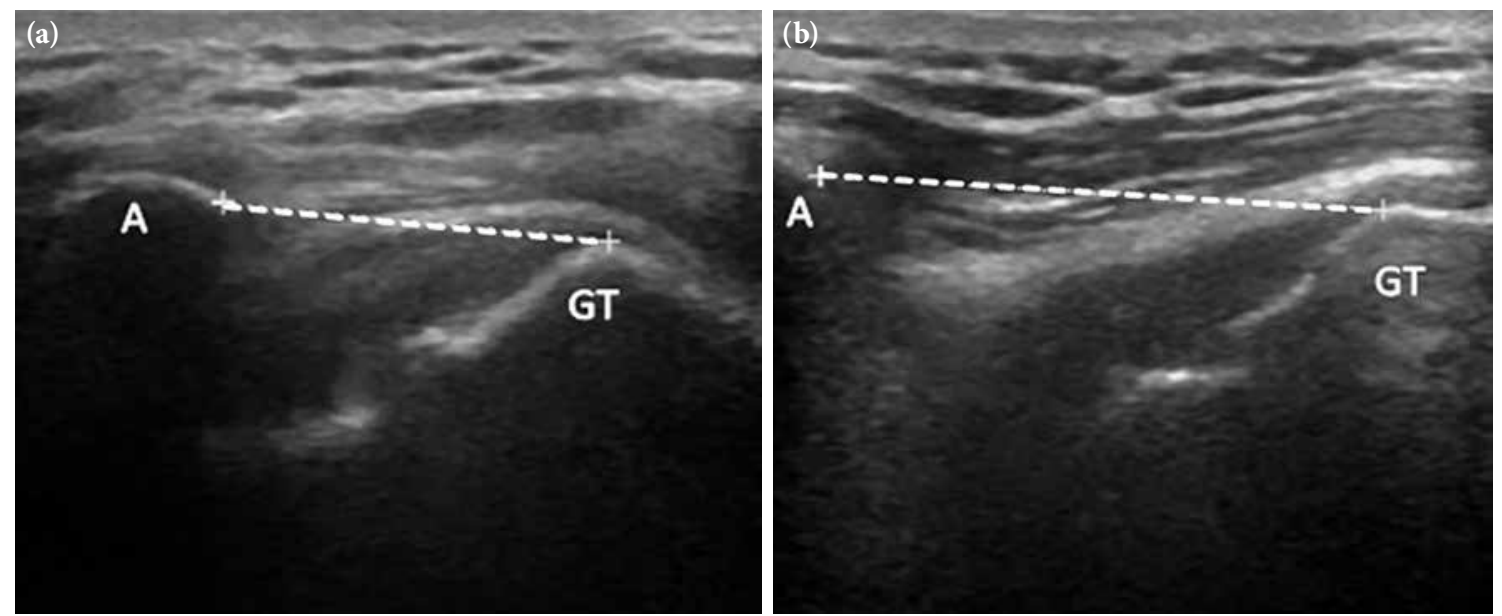

Figure 1. Measurements of acromion (A) to greater tuberosity (GT) (a) at normal side and (b) hemiplegic side.

the acromion process and the nearest margin of the superior part of the greater tuberosity of the humerus were identified on USG imaging, and the distance was measured between these two bony landmarks (Figure 1). ${ }^{[14]}$ For supraspinatus muscle thickness, in longitudinal imaging, the vertical distance between the upper and lower margins of the muscle at scapular notch line was measured. Using this method, the upper trapezius muscle thickness was measured at the most bulky area of the muscle (Figure 2). Posterior deltoid muscle thickness was

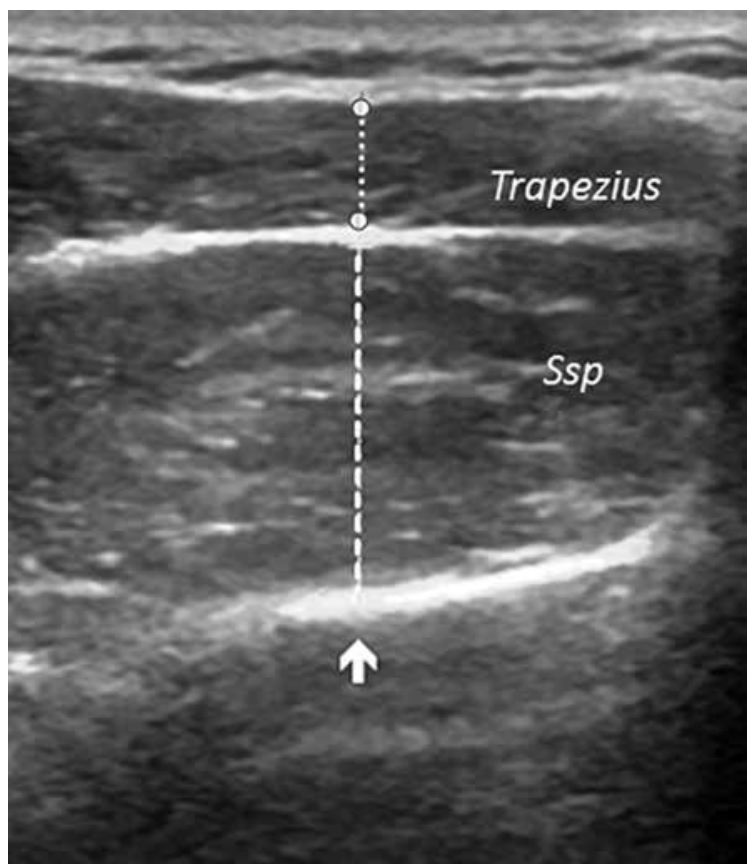

Figure 2. Measurements of upper trapezius and Ssp muscle thicknesses. Arrow indicates a scapular notch. Ssp: Supraspinatus. measured from the vertical distance between the upper and lower limits of the muscle at the glenoid labrum line in axial imaging (Figure 3).

\section{Statistical analysis}

Statistical analysis was performed using the SPSS version 16.0 software (SPSS Inc., Chicago, IL, USA). Data were expressed in mean \pm standard deviation (SD), or median (min-max). The Mann-Whitney $U$ and Fisher's exact tests were used to compare demographic, clinical, and USG variables between the two groups.

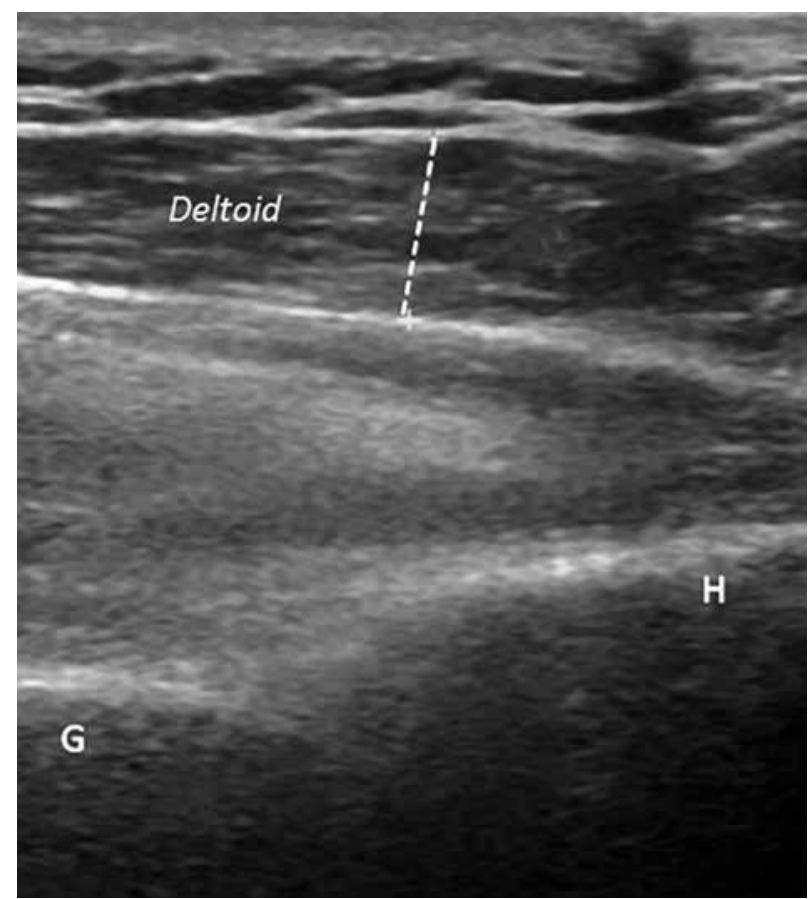

Figure 3. Measurement of posterior deltoid muscle thickness. G; Glenoid; H; Humerus. 
Table 1. Demographic and clinical characteristics of neuromuscular electrical stimulation and control groups

\begin{tabular}{|c|c|c|c|c|c|}
\hline & \multicolumn{2}{|c|}{ NMES group $(\mathrm{n}=12)$} & \multicolumn{2}{|c|}{ Control group $(\mathrm{n}=12)$} & \multirow[b]{2}{*}{$p$} \\
\hline & $\mathrm{n}$ & Mean \pm SD & $\mathrm{n}$ & Mean \pm SD & \\
\hline Age (year) & & $61.5 \pm 10.4$ & & $66.7 \pm 18.1$ & 0.41 \\
\hline Sex & & & & & $0.01^{\star}$ \\
\hline Male & 8 & & 2 & & \\
\hline Female & 4 & & 10 & & \\
\hline Body Mass Index $\left(\mathrm{kg} / \mathrm{m}^{2}\right)$ & & $28.3 \pm 3.7$ & & $28.2 \pm 7.1$ & 0.97 \\
\hline Duration of stroke (month) & & $4.0 \pm 3.3$ & & $3.7 \pm 2.6$ & 0.80 \\
\hline Hemiplegic side & & & & & 0.21 \\
\hline Right & 8 & & 5 & & \\
\hline Left & 4 & & 7 & & \\
\hline
\end{tabular}

The Wilcoxon signed-rank test was used to compare clinical and USG variables between pre- and posttreatment in each group. The Spearman correlation coefficients were used to analyze relationships between the clinical characteristics and USG measurements. A $p$ value of $<0.05$ was considered statistically significant. Post-hoc power of this study was calculated as 0.27 (Clinical program was used for post-hoc analysis, and post-hoc power of A-GT distance was calculated).

\section{RESULTS}

Demographic characteristics of all patients are summarized in Table 1. Age, Body Mass Index, and duration of disease were similar in both groups (for all $\mathrm{p}>0.05$ ). There were 12 patients including eight males and four females in the NMES group and 12 patients including two males and 10 females in the control group, indicating a difference in the sex distribution between the groups $(p=0.01)$. However, the BMRS was not significantly different between the groups: in the NMES group, four patients (33\%) had Stage 2, three patients (25\%) had Stage 3, three patients $(25 \%)$ had Stage 4 , and two patients (17\%) had Stage 5, while in the control group, four patients $(33 \%)$ had Stage 1, five patients $(42 \%)$ had Stage 2, one patient $(8 \%)$ had Stage 4 , and two patients (17\%) had Stage $5(\mathrm{p}=0.08)$.

Clinical characteristics and USG measurements are shown in Table 2. The SDQ index, A-GT distance, and supraspinatus muscle thickness were all improved in the NMES group (for all $\mathrm{p}<0.05$ ). In addition, the VAS-pain scores decreased in both groups (for all $\mathrm{p}<0.05$ ). There was no statistically significant alterations in the other measurements in both groups (for all $\mathrm{p}>0.05$ ). In each group, USG measurements were not found to be correlated with demographic and clinical variables (for all $\mathrm{p}>0.05$ ).
The percentage changes (\%) of clinical and USG measurements are shown in Table 3. The change of A-GT distance was more improved in the NMES group than the control group $(p=0.03)$. The changes of other measurements were not significant statistically (for all $\mathrm{p}>0.05)$.

\section{DISCUSSION}

In the present study, we aimed to determine whether NMES application had any effect on GHS using USG imaging. According to the results of this study, GHS recovered after 20 sessions of NMES treatment. In addition, the SDQ scores decreased in the NMES group, while the VAS-pain scores decreased in both groups.

Previously, positive effects of NMES on GHS in hemiplegic patients were reported in several studies. ${ }^{[15-17,21]}$ A study including 40 acute hemiplegic patients with GHS (20 patients in each control and NMES groups) examined the effects of four-week NMES application to the supraspinatus and posterior deltoid muscles. ${ }^{[21]}$ The authors assessed all patients at four weeks after stroke and, then, at 12 weeks after stroke. They found that NMES application could prevent GHS, although this effect was not sustainable after the end of the treatment. Similarly, in another study, six weeks NMES application decreased GHS in acute hemiplegic patients, although this effect was not sustained after the treatment discontinuation. ${ }^{[16]}$ However, improvement was observed with re-initiation of the NMES treatment. On the other hand, similar effects were not able to be achieved in chronic hemiplegic patients. In another study, the NMES application to the supraspinatus and posterior deltoid muscles in combination with conventional treatment was found to be more beneficial than the conventional treatment alone in hemiplegic GHS patients. ${ }^{[17]}$ 


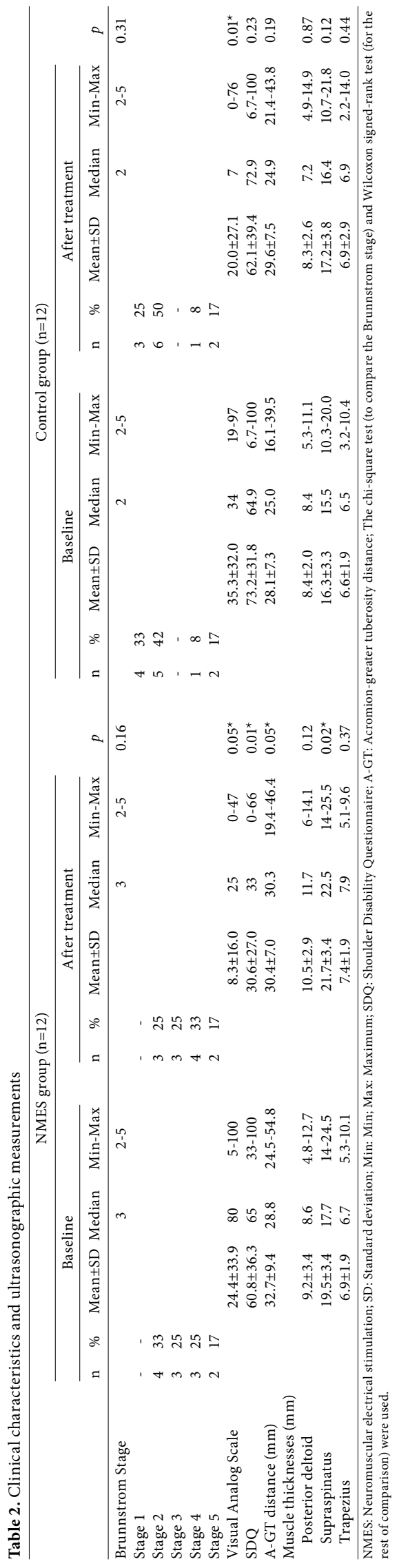

It has been suggested that NMES application to the aforementioned muscles prevents the subluxation prohibiting the inferior displacement of the humeral head. ${ }^{[22]}$ Also, NMES affects agonist and antagonist muscles through local neuronal effects and spinal cord pathways. In the present study, the patients were assessed within 12 months after stroke and, compared to the baseline, 20-session NMES treatment decreased shoulder subluxation. Our findings are also consistent with the literature data. However, to the best of our knowledge, this is the first study to show the NMES effects on GHS in hemiplegic patients using USG. The USG is a useful method for the assessment of GHS in patients with hemiplegic patients.

Furthermore, in the present study, we found that VAS-pain and SDQ scores decreased in the NMES group, while only VAS-pain scores decreased in the control group. However, the percentage changes of the VAS-pain scores did not significantly differ between the NMES and control groups. In the literature, the relationship between GHS and shoulder pain is still controversial. In Cochrane review, although GHS reduced, shoulder pain was reported not to change by NMES treatment. ${ }^{[23]}$ Similarly, in another study, the authors showed that NMES application did not affect shoulder pain, although it reduced shoulder subluxation. ${ }^{[17]}$ Controversially, in another study, the VAS-pain scores were more improved in the NMES treatment group than control group. ${ }^{[24]}$ In this study, we found that, although the SDQ scores decreased in the NMES group, VAS-pain scores decreased in both groups. Also, the percentage change of the VAS-pain scores were not significantly different between the two groups. This can be explained with small sample size of our study.

On the other hand, this study has some limitations. First, power analysis was unable to be performed before the study. Second, our sample size was relatively small. Third, long-term follow-up was unable to be performed. Fourth, there was significant difference in the sex distribution between the two groups due to randomization. Nonetheless, these findings of our study were remarkable.

In conclusion, our study results showed that GHS decreased after 20 sessions of NMES treatment. Based on these findings, USG appears to be a proper imaging tool for the evaluation of GHS in stroke patients. However, further, large-scale, long-term studies are required to confirm these findings. 
Table 3. Percentage changes of clinical characteristics and ultrasonographic measurements

\begin{tabular}{lcccc}
\hline & NMES group $(\mathrm{n}=12)$ & & Control group $(\mathrm{n}=12)$ \\
& Mean \pm SD & & Mean \pm SD & $p$ \\
\hline Visual Analog Scale & $73.3 \pm 36.7$ & $53.1 \pm 30.7$ & 0.30 \\
Shoulder Disability Questionnaire & $38.3 \pm 60.0$ & $12.8 \pm 35.7$ & 0.23 \\
A-GT distance (mm) & $5.7 \pm 10.0$ & $-6.6 \pm 15.8$ & $0.03^{*}$ \\
Muscle thicknesses & & & \\
$\quad$ Posterior deltoid (mm) & $11.7 \pm 22.3$ & $-4.9 \pm 22.4$ & 0.08 \\
$\quad$ Supraspinatus (mm) & $9.7 \pm 13.9$ & $4.5 \pm 12.9$ & 0.35 \\
$\quad$ Trapezius (mm) & $3.9 \pm 23.5$ & $-1.8 \pm 18.3$ & 0.51 \\
\hline
\end{tabular}

NMES: Neuromuscular electrical stimulation; SD: Standard deviation; A-GT: Acromion-greater tuberosity distance.

\section{Declaration of conflicting interests}

The authors declared no conflicts of interest with respect to the authorship and/or publication of this article.

\section{Funding}

The authors received no financial support for the research and/or authorship of this article.

\section{REFERENCES}

1. Stolzenberg D, Siu G, Cruz E. Current and future interventions for glenohumeral subluxation in hemiplegia secondary to stroke. Top Stroke Rehabil 2012;19:444-56.

2. Vuagnat $H$, Chantraine A. Shoulder pain in hemiplegia revisited: contribution of functional electrical stimulation and other therapies. J Rehabil Med 2003;35:49-54.

3. Park GY, Kim JM, Sohn SI, Shin IH, Lee MY. Ultrasonographic measurement of shoulder subluxation in patients with poststroke hemiplegia. J Rehabil Med 2007;39:526-30.

4. Paci M, Nannetti L, Rinaldi LA. Glenohumeral subluxation in hemiplegia: An overview. J Rehabil Res Dev 2005;42:557-68.

5. Kumar P, Kassam J, Denton C, Taylor E, Chatterley A. Systematic review: risk factors for infe-rior shoulder subluxation in patients with stroke. Phys Ther Rev 2010;15:3-11.

6. Boyd EA, Goudreau L, O'Riain MD, Grinnell DM, Torrance GM, Gaylard A. A radiological measure of shoulder subluxation in hemiplegia: its reliability and validity. Arch Phys Med Rehabil 1993;74:188-93.

7. Hayes KW, Sullivan JE. Reliability of a new device used to measure shoulder subluxation. Phys Ther 1989;69:762-7.

8. Van Langenberghe HV, Hogan BM. Degree of pain and grade of subluxation in the painful hemiplegic shoulder. Scand J Rehabil Med 1988;20:161-6.

9. Ikai T, Tei K, Yoshida K, Miyano S, Yonemoto K. Evaluation and treatment of shoulder subluxation in hemiplegia: relationship between subluxation and pain. Am J Phys Med Rehabil 1998;77:421-6.

10. Prévost R, Arsenault AB, Dutil E, Drouin G. Shoulder subluxation in hemiplegia: a radiologic correlational study. Arch Phys Med Rehabil 1987;68:782-5.

11. Berrington de González A, Darby S. Risk of cancer from diagnostic X-rays: estimates for the UK and 14 other countries. Lancet 2004;363:345-51.

12. Tekin L, Kara M, Türker T, Ozçakar L. Shoulder measurements in the early period of ultrasound learning: chasing the butterfly? J Rehabil Med 2011;43:961-2.
13. Ozçakar L, Carli AB, Tok F, Tekin L, Akkaya N, Kara M. The utility of musculoskeletal ultrasound in rehabilitation settings. Am J Phys Med Rehabil 2013;92:805-17.

14. Kumar P, Cruziah R, Bradley M, Gray S, Swinkels A. Intra-rater and inter-rater reliability of ultrasonographic measurements of acromion-greater tuberosity distance in patients with post-stroke hemiplegia. Top Stroke Rehabil 2016;23:147-53.

15. Faghri PD, Rodgers MM, Glaser RM, Bors JG, Ho C, Akuthota P. The effects of functional electrical stimulation on shoulder subluxation, arm function recovery, and shoulder pain in hemiplegic stroke patients. Arch Phys Med Rehabil 1994;75:73-9.

16. Wang RY, Chan RC, Tsai MW. Functional electrical stimulation on chronic and acute hemiplegic shoulder subluxation. Am J Phys Med Rehabil 2000;79:385-90.

17. Koyuncu E, Nakipoğlu-Yüzer GF, Doğan A, Ozgirgin N. The effectiveness of functional electrical stimulation for the treatment of shoulder subluxation and shoulder pain in hemiplegic patients: A randomized controlled trial. Disabil Rehabil 2010;32:560-6.

18. Naghdi S, Ansari NN, Mansouri K, Hasson S. A neurophysiological and clinical study of Brunnstrom recovery stages in the upper limb following stroke. Brain Inj 2010;24:1372-8.

19. Safaz I, Yilmaz B, Yaşar E, Alaca R. Brunnstrom recovery stage and motricity index for the evaluation of upper extremity in stroke: analysis for correlation and responsiveness. Int J Rehabil Res 2009;32:228-31.

20. van der Windt DA, van der Heijden GJ, de Winter AF, Koes BW, Devillé W, Bouter LM. The responsiveness of the Shoulder Disability Questionnaire. Ann Rheum Dis 1998;57:82-7.

21. Linn SL, Granat MH, Lees KR. Prevention of shoulder subluxation after stroke with electrical stimulation. Stroke 1999;30:963-8.

22. Ada L, Foongchomcheay A. Efficacy of electrical stimulation in preventing or reducing subluxation of the shoulder after stroke: a meta-analysis. Aust J Physiother 2002;48:257-67.

23. Price CI, Pandyan AD. Electrical stimulation for preventing and treating post-stroke shoulder pain: a systematic Cochrane review. Clin Rehabil 2001;15:5-19.

24. Chantraine A, Baribeault A, Uebelhart D, Gremion G. Shoulder pain and dysfunction in hemiplegia: effects of functional electrical stimulation. Arch Phys Med Rehabil 1999;80:328-31. 